Reprod. Nutr. Dévelop., 1987, 27 (1 B), 259-260.

\title{
Effet de la nature de la ration, de sa forme de présentation et du niveau d'ingestion sur la vitesse de passage des suppléments protéiques dans le rumen. chez la brebis et chez l'agneau (1)
}

\author{
C. CASTRILlo, J. A. GuAdA, A. VEGA, M. LAINEZ, J. GASA
}

Dpto. Nutrición y Alimentación. Facultad de Veterinaria. Zaragoza. España.

Summary. Fractional outflow rates of chromium treated fish meal were estimated to be $0.0224,0.0311$ and $0.0564 \pm 0.00489 \mathrm{~h}^{-1}$ for mature ewes given a pelleted diet of barley (0.4) and alfalfa hay (0.6) at 1 or $2 \times$ maintenance. With growing lambs fed ad libitum a concentrate pelleted diet diluted with 6 or $23 \%$ of ground barley straw, the rumen outflow rate of protein supplements increased from 0.0480 to $0.0787 \mathrm{~h}^{-1}$ with the level of straw inclusion.

Le temps de séjour des suppléments protéiques dans le rumen est l'un des facteurs qui déterminent la dégradation des constituants azotés, laquelle peut être calculée à partir de la courbe de dégradation in sacco et de la vitesse de passage dans le réticulo-rumen ( $\varnothing$ rskov et Mc Donald, 1979). Celle-ci peut être estimée à partir de l'évolution de la concentration en chrome dans les fécès après l'administration d'une dose unique de supplément mordancé au chrome (Eliman et $\varnothing$ rskov, 1984a).

On a étudié l'effet du niveau alimentaire et de la taille des particules de la ration de base distribuée à des brebis (Expérience 1) et de la proportion de paille dans la ration des agneaux en croissance (Expérience 2) sur la vitesse de passage des suppléments protéiques marqués au chrome à travers le rumen, estimée à partir de l'évolution de la concentration de $\mathrm{Cr}$ dans les fécès.

Matériel et méthodes. Dans l'expérience 1, six brebis adultes ont recu une ration de foin de luzerne et d'orge $(60 / 40)$, broyée $(\varnothing 1 \mathrm{~mm})$ et granulée, couvrant 1 ou 2 fois les besoins d'entretien (G1 et G2) ou la même ration mais avec de la luzerne hachée et l'orge broyée $(\varnothing 3 \mathrm{~mm})$ couvrant 2 fois les besoins d'entretien $(\mathrm{H} 2)$. Les régimes ont été distribués selon un double carré latin $3 \times 3$. Dans l'expérience 2, seize agneaux répartis en deux lots de 4 males et 4 femelles ont reçu ad libitum une ration broyée et granulée à base d'aliments concentrés avec 6 ou $23 \%$ de paille d'orge (régime concentré $C$ et dilué $D$, respectivement).

Après 15 jours d'adaptation à la ration, les animaux de l'expérience 1 ont reçu $50 \mathrm{~g}$ de farine de poisson traitée avec $198 \mathrm{~g} / \mathrm{kg} \mathrm{MS}$ de dichromate de sodium selon la méthode Uden et al. (1980), et des prélèvements de fécès ont été effectués directement dans le rectum à $6,12,18,24,28,32,36,42,48,60,72,96$ et $120 \mathrm{~h}$ après la distribution des produits. La moitié des animaux de l'expérience 2 ont reçu de la farine de poisson marquée et l'autre moitié du tourteau de soja marqué en quantité égale. Les fécès ont été échantillonnées par collecte totale aux mêmes heures que dans l'expérience 1. 
Résultats et discussion. Les ingestions moyennes journalières de matière sèche et les taux de renouvellement moyens estimées à partir des équations de régression des log. 10 de la concentration du chrome dans les fécès (phase descendante) en fonction du temps, sont montrés sur le tableau 1.

TABL. 1. - Influence du régime sur le taux de renouvellement horaire des particules dans le rumen.

\begin{tabular}{|c|c|c|c|c|c|}
\hline \multicolumn{3}{|c|}{ Brebis } & \multicolumn{3}{|c|}{ Agneaux } \\
\hline Régime & $\begin{array}{l}\text { Matière sèche } \\
\text { ingérée } \\
\left(\mathrm{g} / \mathrm{kg} \mathrm{PV} \mathrm{PV}^{75}\right)\end{array}$ & $\begin{array}{c}\text { Taux } \\
\text { de renouvellement } \\
\left(h^{-1}\right)\end{array}$ & Régime & $\begin{array}{c}\text { Matière sèche } \\
\text { ingérée } \\
\left(\mathrm{g} / \mathrm{kg} \mathrm{PV}^{0,75}\right)\end{array}$ & $\begin{array}{c}\text { Taux } \\
\text { de renouvellement } \\
\left(h^{-1}\right)\end{array}$ \\
\hline $\begin{array}{c}G 1 \\
G 2 \\
H 2 \\
\text { e.s. } \bar{x} .(1)\end{array}$ & $\begin{array}{l}32,8 \\
63,6 \\
63,2 \\
1,03\end{array}$ & $\begin{array}{l}0,0224 \\
0,0311 \\
0,0564\end{array}$ & e.s. $\bar{x} \cdot\left({ }^{1}\right)$ & $\begin{array}{l}88,4 \\
85,1\end{array}$ & $\begin{array}{l}0,0480 \\
0,0787\end{array}$ \\
\hline
\end{tabular}

(1) Erreur standard résiduelle.

Le taux de renouvellement a été significativement influencé par la forme de présentation de la ration ( $P<0,01)$. Les différences entre $G 1$ et $G 2$ n'ont pas été statistiquement significatives $(P<0,10)$ mais quand on a estimé le taux de renouvellement à partir de la concentration du marqueur dans le rumen (résultats non publiés) les différences entre les deux niveaux d'alimentation ont été significatives $(P<0,05)\left(0,0270\right.$ et $\left.0,0403 \pm 0,00488 h^{-1}\right)$. Le taux de renouvellement chez les agneaux a été significativement supérieur pour le régime $D(P<0,05)$. On n'a pas trouvé de différences significatives dues au sexe des agneaux ou au type de supplément protéique marqué utilisé. Les résultats sont en concordance avec ceux trouvés par Eliman et Orskov $1984 \mathrm{~b}$ et c) avec des ovins et bovins, respectivement.

Ces variations du taux de renouvellement peuvent influencer considérablement la dégradation des suppléments protéiques. Ainsi, à partir de la courbe de dégradation in sacco du tourteau de soja, déterminée par Orskov et al. (1983) sur des brebis alimentées avec du foin de pré, et suivant la méthode de calcul proposée par Orskov et Mc Donald (1979), on peut estimer une dégradabilité théorique du tourteau de 78,$8 ; 72,8$ et $59,2 \%$ pour les régimes $\mathrm{G} 1, \mathrm{G} 2$ et $\mathrm{H} 2$, respectivement, et de 63,2 et $50,3 \%$ pour les régimes $C$ et $D$. Cependant les variations du taux de renouvellement s'accompagnent souvent de variations de la vitesse de dégradation dont l'effet sur la dégradabilité peut être opposé à l'effet produit par la variation du taux de renouvellement (Gonzalez et al., 1987). II faudrait donc mesurer les deux paramètres dans les mêmes conditions d'alimentation.

Eliman M. E., Orskov E. R., 1984a. Anim. Prod, 39, 77-80.

Eliman M. E., Orskov E. R., 1984b. Anim. Prod., 38, 45-51.

Eliman M. E.. Orskov E. R., 1984c. Anim. Prod., 39, 201-206.

Gonzalez J., Michalet-Doreau B., Poncet C., 1987. Reprod. Nutr. Dévelop., 27, 255-256.

Orskov E. R., Mc Donald I., 1979. J. Agric. Sci., Camb., 92, 499-503.

Orskov E. R., Hughes-Jones M., Eliman M. E., 1983. Livest Pord. Sci., 10, 17-24.

Uden P., Colluci E., Van Soest P. J., 1980. J. Sci. Fd. Agric., 31, 625-632. 